\title{
On extensibility effects in the cross-slot flow bifurcation
}

\author{
Gerardo N. Rocha ${ }^{\mathrm{a}}$, Robert J. Poole ${ }^{\mathrm{b}}$, Manuel A. Alves ${ }^{\mathrm{c}}$, Paulo J. Oliveira ${ }^{\mathrm{d}, *}$ \\ a Depart. Eng. Electromecânica, Unidade Materiais Têxteis e Papeleiros, Universidade da Beira Interior, 6201-001 Covilhã, Portugal \\ b Department of Engineering, University of Liverpool, Brownlow Street, Liverpool L69 3GH, UK \\ ${ }^{c}$ Departamento de Engenharia Química, CEFT, Faculdade de Engenharia da Universidade do Porto, R. Dr Roberto Frias, $4200-465$ Porto, Portugal \\ d Departamento de Engenharia Electromecânica, Unidade Materiais Têxteis e Papeleiros, Universidade da Beira Interior, 6201-001 Covilhã, Portugal
}

\section{A R T I C L E I N F O}

\section{Article history:}

Received 24 April 2008

Received in revised form 18 June 2008

Accepted 19 June 2008

\section{Keywords:}

Cross-slot

Bifurcation

FENE models

Instability predictions

\begin{abstract}
A B S T R A C T
The flow of finite-extensibility models in a two-dimensional planar cross-slot geometry is studied numerically, using a finite-volume method, with a view to quantifying the influences of the level of extensibility, concentration parameter, and sharpness of corners, on the occurrence of the bifurcated flow pattern that is known to exist above a critical Deborah number. The work reported here extends previous studies, in which the viscoelastic flow of upper-convected Maxwell (UCM) and Oldroyd-B fluids (i.e. infinitely extensionable models) in a cross-slot geometry was shown to go through a supercritical instability at a critical value of the Deborah number, by providing further numerical data with controlled accuracy. We map the effects of the $L^{2}$ parameter in two different closures of the finite extendable non-linear elastic (FENE) model (the FENE-CR and FENE-P models), for a channel-intersecting geometry having sharp, "slightly" and "markedly" rounded corners. The results show the phenomenon to be largely controlled by the extensional properties of the constitutive model, with the critical Deborah number for bifurcation tending to be reduced as extensibility increases. In contrast, rounding of the corners exhibits only a marginal influence on the triggering mechanism leading to the pitchfork bifurcation, which seems essentially to be restricted to the central region in the vicinity of the stagnation point.
\end{abstract}

(C) 2008 Elsevier B.V. All rights reserved.

\section{Introduction}

Viscoelastic fluid flow in a cross-slot geometry has been a widely employed test case in computational rheology (e.g. Harlen et al. [1]; Singh and Leal [2]; Remmelgas et al. [3]) because it offers a number of interesting advantages over similar flows. Most significantly it is a geometry that, due to the existence of an interior stagnation point, can generate a high degree of accumulated Hencky strain, much higher than in comparable contraction flows and flow around cylinders, see for instance Peters et al. [4]. Hence it is particularly suited for the investigation of extensional flow properties, allowing almost complete uncoil of flexible polymer molecules. Experimental studies, often in conjuction with numerical simulations (Schoonen et al. [5]), have also been conducted for the cross-slot flow having both sharp and rounded corners, and more sophisticated constitutive models have been employed such as a newly proposed version of the Pom-Pom model (Verbeeten et al.

\footnotetext{
* Corresponding author. Fax: +351 275329972.

E-mail addresses: gerardorocha@portugalmail.pt (G.N. Rocha),

robpoole@liverpool.ac.uk (R.J. Poole), mmalves@fe.up.pt (M.A. Alves),pjpo@ubi.pt
} (P.J. Oliveira).
[6]). Similar interior stagnation-point geometries such as the two(Remmelgas and Leal [7]) and four- (Feng and Leal [8]) roll mills have been considered in numerical simulation studies as a means of obtaining the extensional viscosity of dilute polymer solutions from optical measurements of molecular stretch.

Very recently two papers have been published, one experimental and the other numerical, which have revived interest in the cross-slot geometry. Arratia et al. [9] visualized the flow of a flexible polyacrylamide solution in a microfabricated geometry formed by the crossing of two rectangular cross-section channels and observed that, even for the very low Reynolds number of their experiments, the polymer solution flow tended to evolve to a non-symmetric pattern while the corresponding Newtonian flow remained perfectly symmetric regardless of flow rate. That asymmetric flow pattern, with the incoming flow tending to be preferentially diverted to one of the outlet arms of the cross, was found to be steady at small to moderate Deborah numbers (De), thus corresponding to a supercritical pitchfork bifurcation (Larson [10]). When the flow rate was further increased, the flow remained spatially asymmetric but became unsteady. Such bifurcation phenomena had never been predicted in previous studies concerned with the cross-slot flow, in which most researchers adopted for the solution domain only one quarter of the full geometry, in the belief 

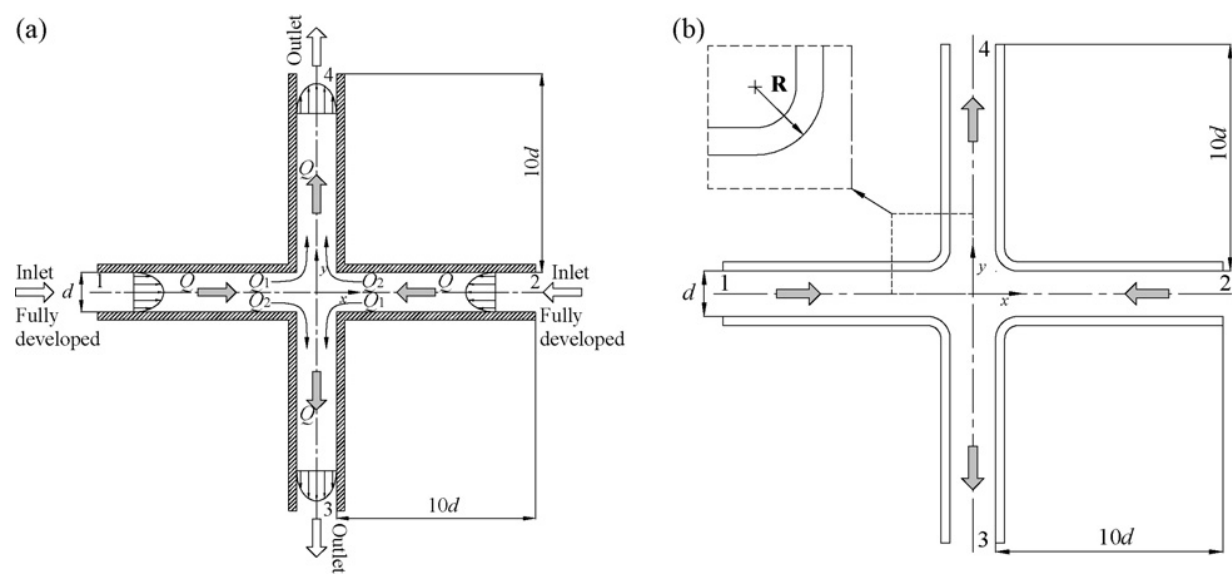

Fig. 1. (a) Schematic of the cross-slot geometry and (b) solution domain for the rounded-corner geometry.

that the resulting flow would follow the symmetry imposed by that same solution domain, and thus sparing useful computational resources. However, motivated by the thought-provoking observations of Arratia et al. [9], Poole et al. [11] simulated the complete cross-slot geometry using the simplest of the available differential constitutive models, the upper-convected Maxwell (UCM) model, and were able to reproduce the main features of the experiments. Their predictions were in qualitative agreement with all the experimental observations: a supercritical bifurcation was found to occur at a critical Deborah number of $D e_{\mathrm{cr}} \approx 0.31$, under creeping-flow conditions, and at higher De the flow became unsteady. A physical explanation was proposed based on a centrifugal-type instability resulting from the distortion of the velocity field as the flow turned into the upper and lower arms, due to the elastic compressive stresses generated by the two opposing incoming fluid streams. In the present study the objective is to probe the effects of finite extensionability, polymer concentration and the effects of corner sharpness. The current results dramatically extend the results of Poole et al., which were based on the essentially phenomenological UCM model, with the use of more realistic finite extendable non-linear elastic (FENE) models. Both the original shear-thinning FENE-P model (Bird et al. [12,13]) and the constant-viscosity FENECR (Chilcott and Rallison [14]) model will be considered.

\section{Problem definition}

The task is to solve the equations of motion, together with a suitable constitutive equation for the stresses, in the two-dimensional geometry depicted in Fig. 1. The two incoming channels are assumed to be aligned with the $x$-axis while the two outgoing channels are aligned with the $y$-axis. All channels have a constant width of $d$, which will serve as a length scale, and are $10 d$ long, a value we have confirmed to be sufficiently large to eliminate end effects. With the $x-y$ origin located at the centre of the cross-slot (Fig. 1), inlet flow conditions with an average velocity $U$, to be used as velocity scale, are prescribed at $x= \pm 10.5 d$, and outlet flow conditions based on zero-streamwise gradients at $y= \pm 10.5 d$.

\subsection{Governing equations}

By assuming incompressibility and separation of stress into a Newtonian solvent contribution, with constant viscosity $\eta_{\mathrm{s}}$, and a non-Newtonian polymeric part $\boldsymbol{\tau}$, the mass and momentum conservation equations are:

$\nabla \cdot \boldsymbol{u}=0$ $\rho \frac{D \boldsymbol{u}}{D t}=-\nabla p+2 \eta_{\mathrm{s}} \boldsymbol{\nabla} \cdot \boldsymbol{D}+\nabla \cdot \boldsymbol{\tau}$,

where $\boldsymbol{D}=1 / 2\left(\nabla \boldsymbol{u}+\nabla \boldsymbol{u}^{\mathrm{T}}\right)$ is the rate of deformation tensor, and the constitutive equations employed are:

$\boldsymbol{\tau}+\lambda\left(\frac{\nabla}{f}\right)=2 \eta_{\mathrm{p}} \boldsymbol{D} \quad($ FENE-CR $)$,

$\boldsymbol{\tau}+\lambda\left(\frac{\nabla}{f}\right)=2 a \eta_{\mathrm{p}}\left(\frac{1}{f}\right) \boldsymbol{D}-a \eta_{\mathrm{p}} \frac{D}{D t}\left(\frac{1}{f}\right) \boldsymbol{I} . \quad$ (FENE-P).

In the above equations $\lambda$ is a relaxation time, $\eta_{\mathrm{p}}$ is the zero-shear rate polymer viscosity (Bird et al. [12] gives $\eta_{\mathrm{p}}=n k T \lambda b /(b+5)$ in terms of basic microstructure quantities), the constant $a$ is defined as $a=L^{2} /\left(L^{2}-3\right)$ where $L^{2}$ (or, equivalently, $b$ ) is the extensibility parameter, $f$ is a function of the stress invariants defined below, $\boldsymbol{I}$ is the identity tensor, and $(\mathbf{A})=D \mathbf{A} / D t-\left(\mathbf{A} \cdot \nabla u+\nabla u^{\mathrm{T}} \cdot \mathbf{A}\right)$ denotes the upper-convected derivative (Oldroyd [15]). Zero-shear rate viscosities are often prescribed by a solvent viscosity ratio defined as $\beta=\eta_{\mathrm{s}} / \eta_{0}$, where $\eta_{0}=\eta_{\mathrm{s}}+\eta_{\mathrm{p}}$ is the zero-shear rate viscosity. For both models, shear-thinning in the first-normal stress coefficient $\Psi_{1}$ is controlled by the function $f$ which depends on the trace of the polymer stress as

$f(\tau)=\frac{L^{2}+\left(\lambda / \eta_{\mathrm{p}}\right) \operatorname{Tr}(\boldsymbol{\tau})}{L^{2}-3} \quad($ FENE-CR $)$,

and

$f(\tau)=\frac{L^{2}+\left(\lambda / a \eta_{\mathrm{p}}\right) \operatorname{Tr}(\boldsymbol{\tau})}{L^{2}-3} \quad($ FENE-P $)$.

These two functions have an almost identical dependence on the trace of the stress, except for the factor $a$ which is approximately unity when $L^{2}$ is large $(L \rightarrow \infty, a \rightarrow 1)$. In spite of this agreement, the shear viscosity dependency of the FENE-P model is markedly different as can be inferred from inspection of the first term on the right hand side of Eq. (4) because $\eta_{\mathrm{p}}$ is divided by a term proportional to $\operatorname{Tr}(\boldsymbol{\tau})$ thus inducing shear-thinning of the shear viscosity.

We recall that the FENE-P model was derived from kinetic theory by Bird et al. [13] for a two-bead/spring-connector molecular model, without accounting for dumbbell interactions and excluded volume effects, and the only approximation required to obtain the above closed-form equation was the Peterlin approximation for the connector force. The model predicts a shear-thinning shear viscosity but since in the present calculations the minimum value of the solvent viscosity ratio is just $\beta=0.05$, the decay of $\eta(\dot{\gamma})$ is at most about one decade. In contrast the FENE-CR model results 

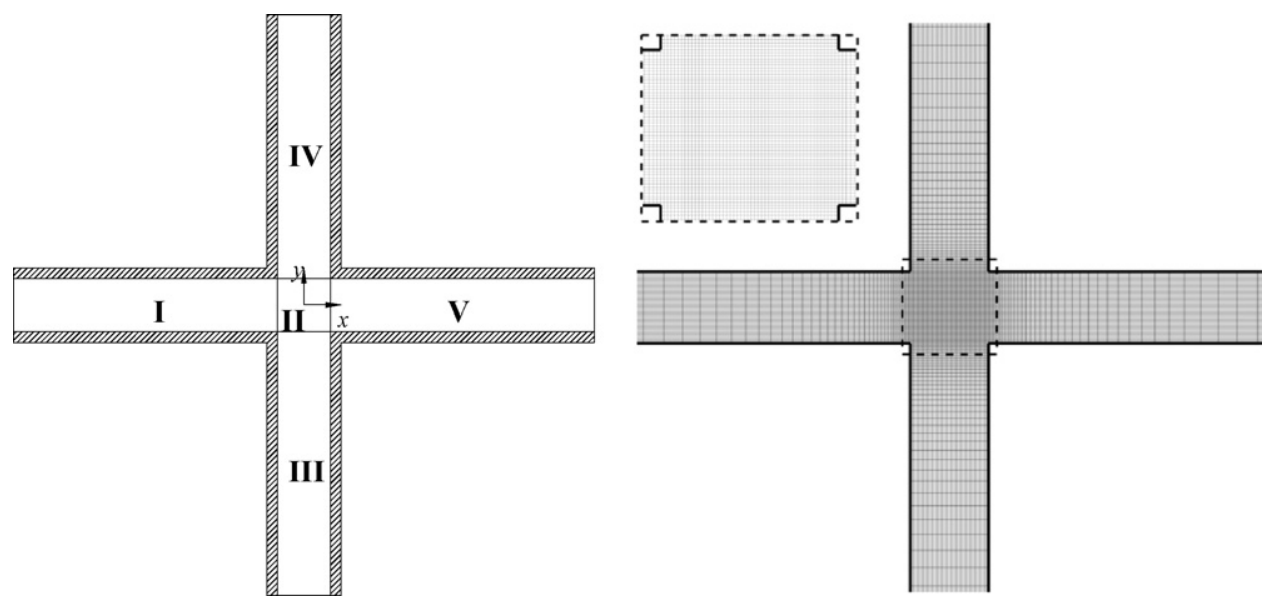

Fig. 2. Mesh-generating blocks and detail of mesh near the intersection $(-4 \leq x / d, y / d \leq 4)$.

from an empirical a-posteriori modification to the FENE-P equation in order to produce a model which exhibits a constant shear viscosity (Chilcott and Rallison [14]). In spite of this somewhat adhoc empiricism, the model is very useful as it allows the study of elastic effects without the sometimes conflicting influence of shear-thinning in shear viscosity. In both models the molecular extensibility is measured by the parameter $L^{2}$ which represents the square of the ratio of the maximum dumbbell extension to its equilibrium value. Finally we note that Eqs. (4) and (6) already include the corrections introduced by Bird et al. [12] to their original FENE$P$ equation in order to make it more consistent, and the relation between $L^{2}$ and the more common $b$ parameter is:

$L^{2}=b+5$.

With this correspondence, the parameter $a$ can also be defined as $a=(b+5) /(b+2)$.

\subsection{Solution method}

A finite-volume method (Oliveira et al. [16]) is used to solve the set of Eqs. (1)-(3), or (1), (2) and (4) depending on the choice of FENE model. The scheme is a fully implicit, time-marching, pressurecorrection method, based on the collocated mesh arrangement, with all unknown variables (velocities, pressure and stresses) calculated at the centre of the control-volumes, and employing second-order discretization schemes: the three-time level method for the unsteady terms; the CUBISTA scheme (Alves et al. [17]) for the advective terms; and central differences for the diffusive and source terms. The algorithm was here applied as described in Oliveira [18] where further details can be found.

The solution domain of Fig. 1 was covered with a computational mesh generated by blocks, as shown in Fig. 2, and the main mesh characteristics are provided in Table 1 . This base mesh was designed after a number of mesh refinement studies so that we guarantee that the results were only marginally affected by mesh fineness:

Table 1

Main characteristics of base mesh

\begin{tabular}{lllll}
\hline Block & $N_{x}$ & $N_{y}$ & $f_{x}$ & $f_{y}$ \\
\hline I & 50 & 51 & 0.929296 & 1.0 \\
II & 51 & 51 & 1.0 & 1.0 \\
III & 51 & 50 & 1.0 & 0.929296 \\
IV & 51 & 50 & 1.0 & 1.075369 \\
V & 50 & 51 & 1.075369 & 1.0 \\
NCV & & & & \\
\hline
\end{tabular}

some results of these studies are provided later in order to quantify the accuracy of the predictions. It is worth noting that we intentionally selected the number of cells placed uniformly across each channels to be an odd figure (51 cells, giving a nominal cell spacing of $\Delta y \cong 0.02 d$ ) thus producing a row of cells exactly along the centreline of the two intersecting channels. Although orthogonal, the mesh is non-uniform along the length of the inlet and outlet channels in order to concentrate cells in the common intersection zone of the cross-slot (block II in Fig. 2).

For the FENE-CR model, on account of the constant shear viscosity, the fully developed velocity profile is parabolic as in the Newtonian case and it is a relatively straightforward matter (e.g. Oliveira [19]) to derive the shear $\tau_{x y}$ and normal $\tau_{x x}$ stress component profiles to be applied at the two inlet sections. For the FENE-P model, and especially when $\beta \neq 0$, the problem of obtaining fully developed velocity and stress profiles is much more involved and the analytical solution obtained by Cruz et al. [20] was applied at the inlet sections. With these exact profiles imposed at inlet it is far simpler to evaluate the pressure loss due to the cross-slot (Section 3.5) since a linearly decaying pressure variation is established immediately from the two inlet planes.

\section{Results}

Quantitative results are essentially provided in terms of a bifurcation variable, which we have chosen to be the flow rate imbalance $D Q$ as a function of the Deborah number, $D e=\lambda U / d$. A robust bifurcation variable should vary from 0 , indicating absence of bifurcation, to \pm 1 above the critical point when three steady solutions are possible (the "symmetric" solution, which is not stable; a bifurcated solution with more flow coming from the left and going through the upper channel; and the opposite bifurcated solution with more flow from the left into the lower channel). The definition is thus $D Q=\left(Q_{1}-Q_{2}\right) / Q$ with flow rates $Q_{1}$ and $Q_{2}$ indicated in Fig. 1; the total flow rate in each incoming channel is $Q=U d$ and this incoming flow splits at the cross-slot region such that $Q=Q_{1}+Q_{2}$.

Most calculations are for a Reynolds number $\left(\operatorname{Re}=\rho U d / \eta_{0}\right)$ of exactly zero, since it has already been established that the instability is purely elastic in nature and we do not want to consider inertial effects here; this was specified by omitting the convective terms in the momentum equations $(\rho \boldsymbol{u} \cdot \nabla \boldsymbol{u}=\mathbf{0})$ but, of course, not in the constitutive equations. Poole et al. [11] have shown that a small increase of inertia, with Re being increased from 0 to 3, leads to an attenuation of the asymmetry. Simulations containing a small, but 


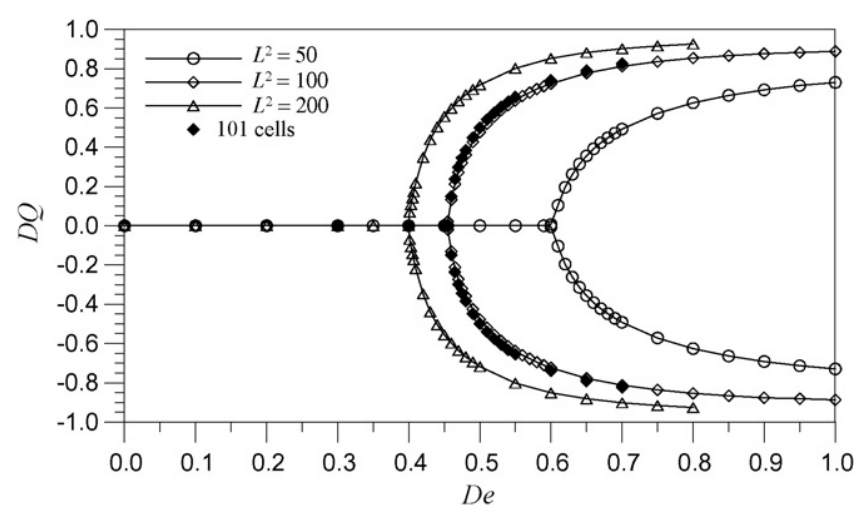

Fig. 3. Variation of asymmetry parameter $D Q$ with Deborah number for the FENE-CR model: influence of extensibility $(\beta=0.1)$. Symbols $(\bullet)$ : finer mesh with $L^{2}=100$.

Table 2

Bifurcation data for the FENE-CR fluid: (a) varying $L^{2}(\beta=0.1)$ and (b) varying $\beta$ $\left(L^{2}=100\right)$ (critical conditions indicated in bold)

\begin{tabular}{|c|c|c|c|c|c|}
\hline \multicolumn{2}{|c|}{ (a) $L^{2}=\mathbf{5 0}$} & \multicolumn{2}{|c|}{$L^{2}=\mathbf{1 0 0}$} & \multicolumn{2}{|c|}{$L^{2}=\mathbf{2 0 0}$} \\
\hline De & $D Q$ & De & $D Q$ & De & $D Q$ \\
\hline 0.00 & 0.000 & 0.00 & 0.000 & 0.00 & 0.000 \\
\hline 0.10 & 0.000 & 0.10 & 0.000 & 0.10 & 0.000 \\
\hline 0.20 & 0.000 & 0.20 & 0.000 & 0.20 & 0.000 \\
\hline 0.30 & 0.000 & 0.30 & 0.000 & 0.30 & 0.000 \\
\hline 0.40 & 0.000 & 0.40 & 0.000 & 0.40 & 0.070 \\
\hline 0.50 & 0.000 & 0.455 & 0.021 & 0.41 & 0.218 \\
\hline 0.60 & 0.006 & 0.46 & 0.132 & 0.42 & 0.348 \\
\hline 0.61 & 0.104 & 0.47 & 0.272 & 0.43 & 0.438 \\
\hline 0.62 & 0.196 & 0.48 & 0.360 & 0.44 & 0.504 \\
\hline 0.63 & 0.262 & 0.49 & 0.426 & 0.45 & 0.556 \\
\hline 0.64 & 0.314 & 0.50 & 0.478 & 0.46 & 0.598 \\
\hline 0.65 & 0.356 & 0.51 & 0.520 & 0.47 & 0.636 \\
\hline 0.66 & 0.392 & 0.52 & 0.556 & 0.48 & 0.668 \\
\hline 0.67 & 0.422 & 0.53 & 0.588 & 0.49 & 0.694 \\
\hline 0.68 & 0.448 & 0.54 & 0.614 & 0.50 & 0.718 \\
\hline 0.69 & 0.472 & 0.55 & 0.638 & 0.55 & 0.802 \\
\hline 0.70 & 0.492 & 0.60 & 0.724 & 0.60 & 0.852 \\
\hline 0.75 & 0.572 & 0.70 & 0.812 & 0.65 & 0.882 \\
\hline 0.80 & 0.626 & 0.80 & 0.854 & 0.70 & 0.902 \\
\hline 0.90 & 0.692 & 0.90 & 0.876 & 0.75 & 0.916 \\
\hline 1.00 & 0.730 & 1.00 & 0.888 & 0.80 & 0.926 \\
\hline \multicolumn{2}{|c|}{ (b) $\beta=\mathbf{0 . 0 5}$} & \multicolumn{2}{|c|}{$\beta=0.10$} & \multicolumn{2}{|c|}{$\beta=0.20$} \\
\hline De & $D Q$ & De & $D Q$ & De & $D Q$ \\
\hline 0.00 & 0.000 & 0.00 & 0.000 & 0.00 & 0.000 \\
\hline 0.10 & 0.000 & 0.10 & 0.000 & 0.10 & 0.000 \\
\hline 0.20 & 0.000 & 0.20 & 0.000 & 0.20 & 0.000 \\
\hline 0.30 & 0.000 & 0.30 & 0.000 & 0.30 & 0.000 \\
\hline 0.40 & 0.002 & 0.40 & 0.000 & 0.40 & 0.000 \\
\hline 0.41 & 0.190 & 0.455 & 0.021 & 0.50 & 0.000 \\
\hline 0.42 & 0.316 & 0.46 & 0.132 & 0.60 & 0.000 \\
\hline 0.43 & 0.398 & 0.47 & 0.272 & 0.65 & 0.000 \\
\hline 0.44 & 0.460 & 0.48 & 0.360 & 0.67 & 0.014 \\
\hline 0.45 & 0.510 & 0.49 & 0.426 & 0.68 & 0.056 \\
\hline 0.46 & 0.552 & 0.50 & 0.478 & 0.69 & 0.198 \\
\hline 0.47 & 0.586 & 0.51 & 0.520 & 0.70 & 0.296 \\
\hline 0.48 & 0.616 & 0.52 & 0.556 & 0.71 & 0.366 \\
\hline 0.49 & 0.642 & 0.53 & 0.588 & 0.72 & 0.420 \\
\hline 0.50 & 0.666 & 0.54 & 0.614 & 0.73 & 0.446 \\
\hline 0.55 & 0.748 & 0.55 & 0.638 & 0.74 & 0.502 \\
\hline 0.60 & 0.798 & 0.60 & 0.724 & 0.75 & 0.534 \\
\hline 0.65 & 0.830 & 0.70 & 0.812 & 0.80 & 0.644 \\
\hline 0.70 & 0.852 & 0.80 & 0.854 & 0.85 & 0.710 \\
\hline- & - & 0.90 & 0.876 & 0.90 & 0.752 \\
\hline - & - & 1.00 & 0.888 & 1.00 & 0.804 \\
\hline
\end{tabular}

Table 3

Convergence with mesh refinement: values of $D Q$ for $L^{2}=100$ and $\beta=0.1$

\begin{tabular}{llllll}
\hline De & $\begin{array}{l}\text { Mesh 1 } \\
N=51\end{array}$ & $\begin{array}{l}\text { Mesh 2 } \\
N=101\end{array}$ & Extrapolation & Error Mesh 1 (\%) & Error Mesh 2(\%) \\
\hline 0.5 & 0.478 & 0.499 & 0.506 & 5.5 & \\
0.6 & 0.724 & 0.739 & 0.744 & 2.7 & 0.7 \\
0.7 & 0.812 & 0.825 & 0.829 & 2.0 & 0.5 \\
\hline
\end{tabular}

finite, amount of inertia $(R e=0.01)$ where essentially identical to the creeping-flow results $(\operatorname{Re}=0)$.

\subsection{Effect of extensibility}

Fig. 3 shows bifurcation plots obtained with the FENE-CR model for three values of the extensibility parameter, $L^{2}=50,100$ and 200 , at a fixed base value of the solvent viscosity ratio $\beta=0.1$. For the purpose of future comparisons, the corresponding data are detailed in Table $2 \mathrm{a}$. $L^{2}=100$ is a typical extensibility value which has been used in many other studies (Bird et al. [13]; Remmelgas et al. [3]; Oliveira [18]) and so here we are doubling and halving that base extensibility. It is also useful to keep in mind that the UCM and Oldroyd-B models correspond to $L^{2}=\infty$ and the critical Deborah number for the occurrence of a bifurcated solution was found to be $D e_{\mathrm{cr}} \approx 0.31$ by Poole et al. [11] for the UCM model. The variation of the asymmetry parameter, $D Q$ vs. De seen in Fig. 3 is typical of supercritical pitchfork bifurcations, with two stable (and steady) possible end states; either most of the flow entering from the left channel leaves through the upper vertical channel $(D Q \rightarrow+1)$ or, alternatively, most flow leaves through the lower channel $(D Q \rightarrow-1)$. Numerically, any of theses two solutions may be obtained, depending on the exact initial conditions, time step employed, or any other "numerical" parameter. The bifurcation is thus very similar to the viscoelastic Coanda effect predicted in two-dimensional planar expansions (Oliveira [19]; Rocha et al. [21])-compare Fig. 3 here with Fig. 13 in Oliveira [19] (for a 1:3 expansion) for example. However an important difference should be pointed out: while in the planar expansion the pitchfork bifurcation was essentially an inertial effect (for increasing $R e$ ) with elasticity tending to promote stability, here for the cross-slot it is a purely elastic instability $(R e=0)$ and elasticity through extensional viscosity $\left(\eta_{\mathrm{E}}\right.$ increases with $\left.L^{2}\right)$ having a destabilising effect. When the molecular stretch is allowed to reach large values, thus inducing strong extensional viscosities in the cross-slot central region where polymer molecules tend to remain for a long time and therefore are expected to uncoil and become fully stretched, the bifurcation occurs at lower flow rates (that is, as $L^{2}$ increases, then $D e_{\mathrm{cr}}$ decreases, cf. Fig. 11 further down).

As commented upon above, in order to quantify the discretization errors involved in our simulations we have carried out a set of simulations for the case $L^{2}=100$ using a mesh with double the number of control volumes along each direction (having a total number of 50,601 control volumes and a minimum mesh spacing of $\Delta y \cong 0.01 d$ ) and these predictions are also indicated in Fig. 3 . In the figures the differences between meshes are hardly perceptible and we may conclude that our base mesh provides adequate accuracy. Predictions of $D Q$ on the two meshes at some selected Deborah numbers are also provided in Table 3, indicating differences between meshes of at most $5 \%$ in the region close to the critical point when $D Q$ raises sharply.

Theoretically, a bifurcation variable such as $D Q$ should scale as $D Q \sim \sqrt{D e-D e_{\mathrm{cr}}}$ for $D e$ above the critical point, $D e_{\mathrm{cr}}$; this scaling is apparent from Fig. 3 and will be discussed in the next subsection, where both influences of $L^{2}$ and $\beta$ are analysed. 

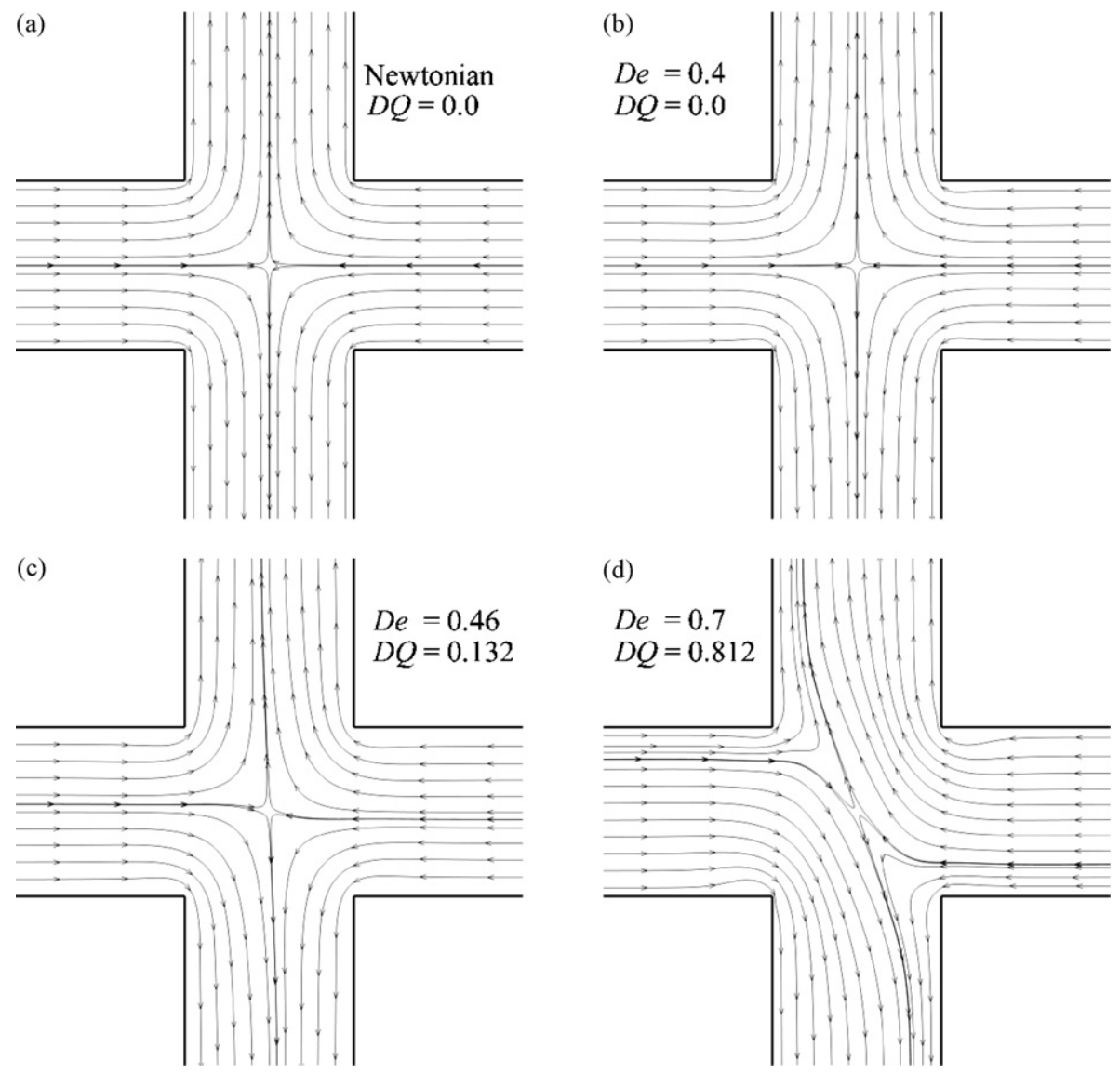

Fig. 4. Streamline maps for: (a) Newtonian fluid; (b) $D e=0.40$; (c) $D e=0.46$; and (d) $D e=0.70$ (FENE-CR, $L^{2}=100$ and $\beta=0.1$ ).

Streamline plots provide a clear visualization of the bifurcated flow, allowing us to contrast the final flow pattern against the symmetrical flow of the Newtonian case (which can be thought as the solvent without any added polymer solute), and also for observing how the degree of bifurcation increases rapidly over a short range of $D e$ immediately above the critical value. Fig. 4 shows a zoomed view of streamlines for the base case $\left(L^{2}=100, \beta=0.1\right)$ at four Deborah numbers, namely $D e=0$ (Newtonian), 0.4 (below critical), 0.46 (immediately above critical) and 0.70 (almost fully bifurcated), thus illustrating the previous comments. The corresponding stress fields $\tau_{x x}$ and $\tau_{y y}$, normalised using a viscous scaling, are shown in Figs. 5 and 6 for increasing De: these evolve from the centrally symmetrical situation of Newtonian flow - in the absence of convective terms, since $R e=0$, the equations of motion are linear and symmetry about both $x$ and $y$ axis must be satisfied (and also about the two diagonals, $y= \pm x$ ) - to the compressive stresses for the viscoelastic cases along the horizontal channels, and the typical "birefringent strands" due to the strong and localised extensional flow along the centrelines of the vertical channels. These birefringence strands (Harlen et al. [1]) have been the focus of much research in previous studies concerned with the cross-slot geometry, in particular researchers have used constant-viscosity models, and modifications to existing ones, in order to predict the longer strands observed in experiments (Remmelgas et al. [3]). However, the birefringent strands do not appear to be related to the origin of the bifurcation, which is rather linked to the compressive flow in the inlet channels (Poole et al. [11]). It should be pointed out that molecular stretch is proportional to the trace of the polymer stress tensor, but a plot of $\tau_{x x}+\tau_{y y}$ is essentially unchanged from the $\tau_{y y}$ contour plots of Fig. 6 and so is not included here.

\subsection{Effect of concentration}

It is possible to define a polymer concentration parameter $c$ in terms of the solvent viscosity ratio $\beta$, as was done most notably by Leal and co-workers (Singh and Leal [2]; Remmelgas et al. [3] and [7]; and Feng and Leal [8]); thus $c=\eta_{\mathrm{p}} / \eta_{\mathrm{s}}$ which implies the relation $c=(1 / \beta)-1$. Therefore Boger fluids, whose shear-thinning in shear viscosity is masked by the dominant solvent viscosity, should have values of $\beta$ close to 1 and hence a small concentration parameter $c$, while by decreasing $\beta$ from the Newtonian value of unity, the concentration parameter increases up to infinity. It is therefore expected that as $\beta$ decreases, elasticity effects should become more dominant and the behaviour of $D Q$ vs. De in terms of diminishing $\beta$ should replicate qualitatively the conclusions obtained in the previous subsection for increasing $L^{2}$. This situation is indeed the case as shown by Fig. 7 (numerical data provided in Table 2b), cf. Fig. 3, but an important distinction should be made: while $L^{2}$ is closely related to elasticity through extensional effects, $\beta$ is related to elasticity through normal-stress difference $\left(N_{1}\right)$ effects in shear It should therefore be possible to separate the two effects by incorporating the effect of $\beta$ in a re-scaled Deborah number, for example $D e \leftarrow D e(1-\beta)$, and thus completely isolate extensional elasticity and normal-stresses elasticity. However, FENE models do not allow a clear separation of these two influences and therefore re-scaling the Deborah number does not translate into a complete collapse of elasticity effects, although the main influence is still captured. Such duality is present in the bifurcation scaling (cf. Fig. 11b in Section 3.3):

$D Q \propto A\left(L^{2}\right) \sqrt{D e-D e_{\mathrm{cr}}\left(\beta, L^{2}\right)}$, 
(a)

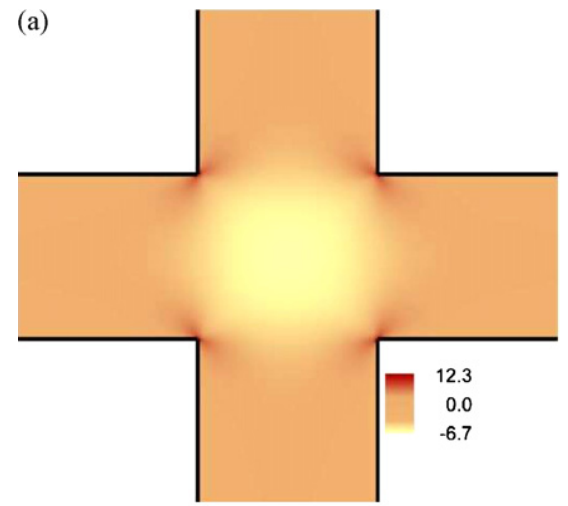

(c)

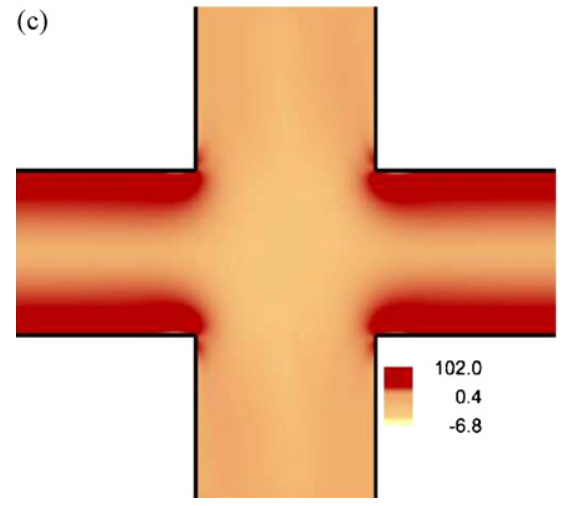

(b)

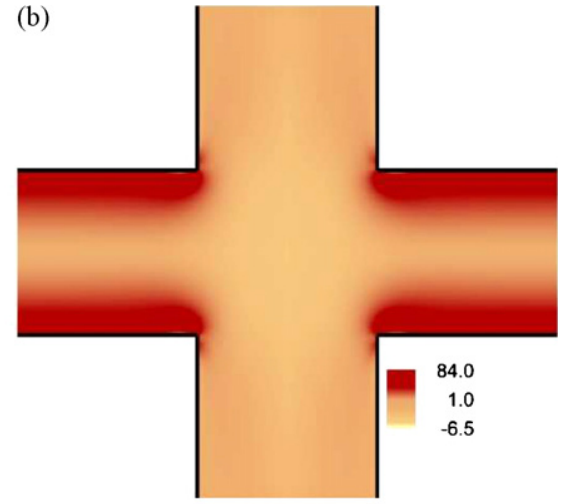

(d)

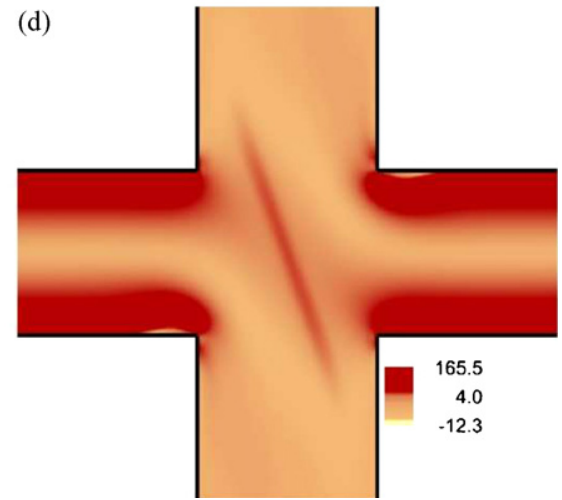

Fig. 5. Contour plots of horizontal normal stress $\tau_{x x} /\left(\eta_{0} U / d\right)$ for: (a) Newtonian fluid; (b) $D e=0.40$; (c) $D e=0.46$; and (d) $D e=0.70$. (FENE-CR, $L^{2}=100$ and $\beta=0.1$ ).

(a)

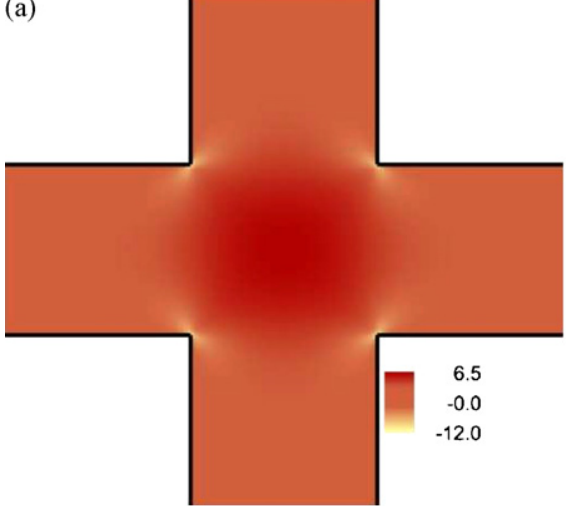

(c)

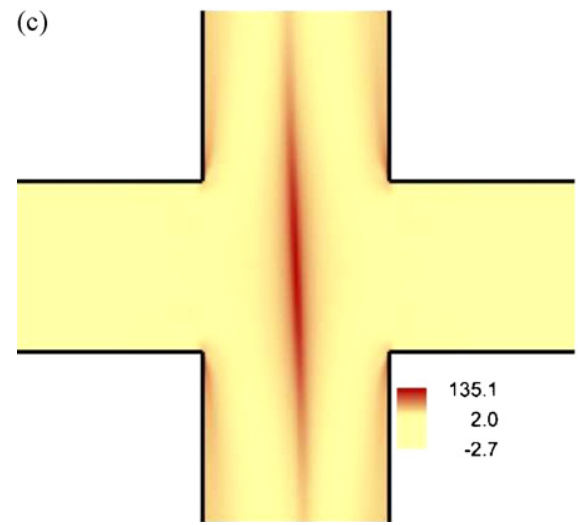

(b)

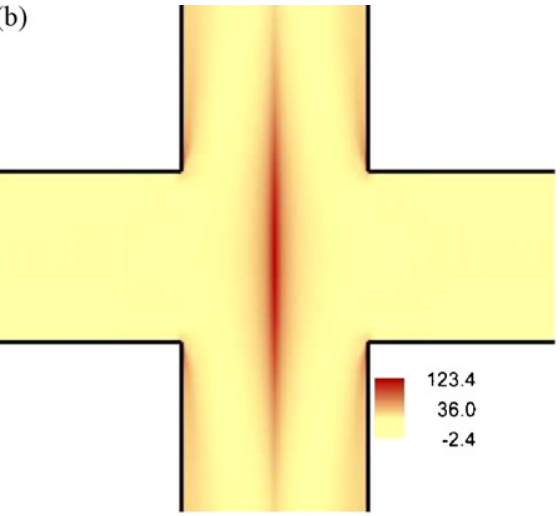

(d)

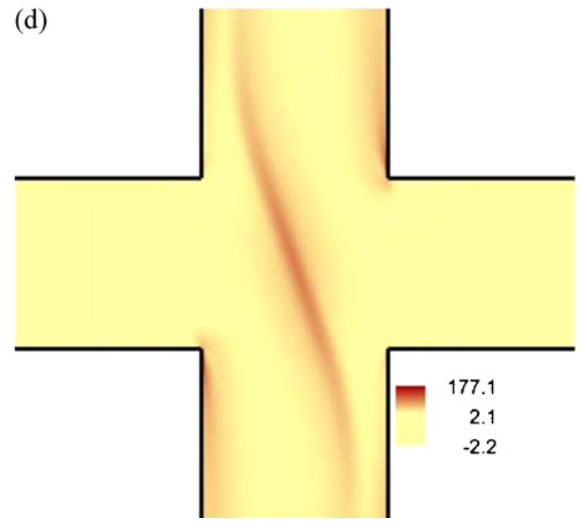

Fig. 6. Contour plots of vertical normal stress $\tau_{y y} /\left(\eta_{0} U / d\right)$ for: (a) Newtonian fluid; (b) $D e=0.40$; (c) $D e=0.46$; and (d) $D e=0.70$. (FENE-CR, $L^{2}=100$ and $\beta=0.1$ ). 


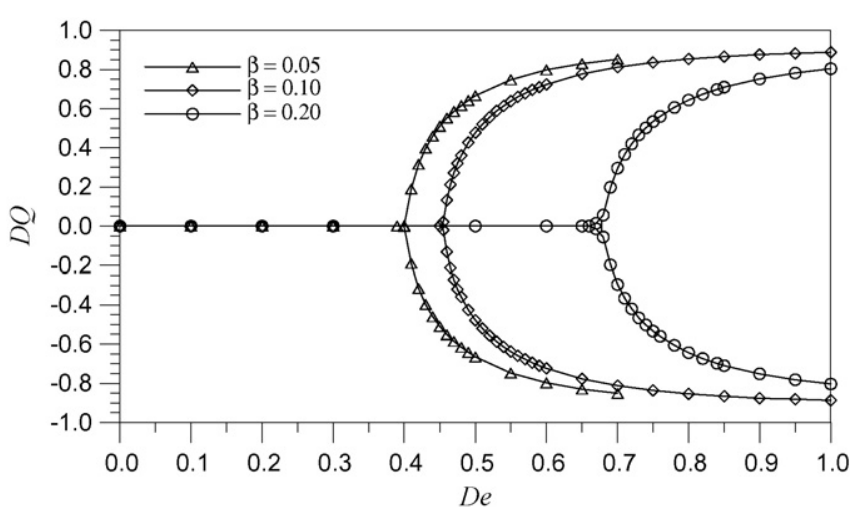

Fig. 7. Variation of asymmetry parameter $D Q$ with Deborah number for the FENE$\mathrm{CR}$ model: influence of polymer concentration through solvent viscosity ratio $\beta$ $\left(L^{2}=100\right)$.

which is drawn on the basis of Figs. 3 and 7. While the critical Deborah number is controlled by both normal stress and extensional elasticity, the magnitude of the bifurcation is mainly controlled by extensibility. Our predictions yield the results for $A$ and $D e_{\mathrm{cr}}$ given in Tables 4 and 5 which also include the results for the rounded corner cases which we discuss in detail in the following section. While in Table 4 the critical De is obtained directly from the raw data of Table 1, being thus dependent on the step used to increase $D e$, in Table 5 both $A$ and $D e_{\mathrm{cr}}$ are estimated from a linear correlation between $D Q^{2}$ and $D e$ close to the critical $D e$.

Figure 8 provides an illustration of the quality of the fitting defined by Eq. (8) when most data points of the $D Q$ vs. De variation are included and the $D e_{\mathrm{cr}}$ are assumed from the data of Table 2 and are given in Table 4. A fine tuning of a correlation like Eq. (8) is achieved by linearising it to obtain $D Q^{2}=A^{2}\left(D e-D e_{\mathrm{cr}}\right)$ and thus arrive at $A$ and $D e_{\mathrm{cr}}$ by a standard linear regression technique. In this way the critical point in terms of Deborah number is not based on the assumed step for the increase in De, which was the same for all

Table 4

Critical Deborah numbers for bifurcation: (a) varying $L^{2}$, at $\beta=0.1$ and (b) varying $\beta$, at $L^{2}=100$

\begin{tabular}{clll}
\hline & Sharp & $R=0.05 d$ & $R=0.5 d$ \\
\hline (a) $L^{2}$ & & & \\
50 & 0.61 & 0.63 & 0.85 \\
100 & 0.46 & 0.47 & 0.62 \\
200 & 0.41 & 0.42 & 0.54 \\
& & & \\
(b) $\beta$ & & & 0.56 \\
0.05 & 0.41 & 0.42 & 0.62 \\
0.10 & 0.46 & 0.47 & 0.86 \\
0.20 & 0.68 & 0.70 & \\
\hline
\end{tabular}

\section{Table 5}

Parameters of the square-root fitting according to Eq. (8): (a) varying $L^{2}(\beta=0.1)$ and (b) varying $\beta\left(L^{2}=100\right)$

\begin{tabular}{|c|c|c|c|c|c|c|}
\hline & \multicolumn{2}{|c|}{ Sharp corners } & \multicolumn{2}{|c|}{$R=0.05 d$} & \multicolumn{2}{|c|}{$R=0.5 d$} \\
\hline & $D e_{\mathrm{cr}}$ & $A$ & $D e_{\mathrm{cr}}$ & $A$ & $D e_{\mathrm{cr}}$ & $A$ \\
\hline \multicolumn{7}{|c|}{ (a) $L^{2}(\beta=0.1)$} \\
\hline 50 & 0.607 & 1.74 & 0.625 & 1.69 & 0.849 & 1.34 \\
\hline 100 & 0.456 & 2.32 & 0.468 & 2.26 & 0.618 & 1.80 \\
\hline 200 & 0.402 & 2.58 & 0.410 & 2.47 & 0.539 & 2.11 \\
\hline \multicolumn{7}{|c|}{ (b) $\beta\left(L^{2}=100\right)$} \\
\hline 0.05 & 0.402 & 2.36 & 0.412 & 2.31 & 0.554 & 1.93 \\
\hline 0.10 & 0.456 & 2.32 & 0.468 & 2.26 & 0.618 & 1.80 \\
\hline 0.20 & 0.682 & 2.18 & 0.698 & 2.11 & 0.855 & 1.61 \\
\hline
\end{tabular}
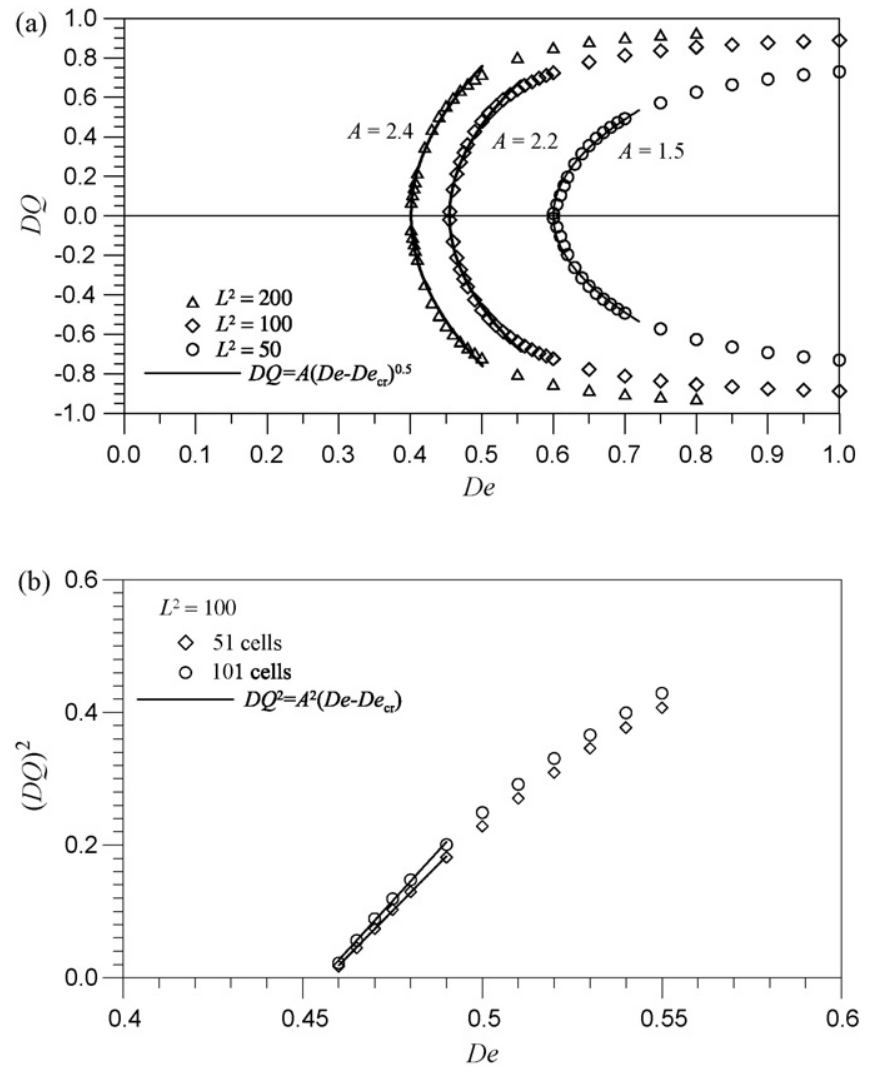

Fig. 8. (a) Square-root fitting of the bifurcation data for the FENE-CR model with three extensibility values (cf. Eq. (8)) and (b) Zoomed view shows $D Q^{2}$ vs. De on two meshes including linear regressions.

meshes (we varied $D e$ by steps of $\Delta D e=0.01$ ), but rather on the particular correlation defined as $D Q \approx \sqrt{D e-D e_{\mathrm{cr}}}$ valid for pitchfork bifurcations as discussed in the previous subsection. By employing a standard least-squares fitting procedure we obtained for the base case $\left(L^{2}=100, \beta=0.1\right)$ the values $A=2.344$ and $D e_{\mathrm{cr}}=0.457$ (linear correlation coefficient, $r=0.999)$ on the base mesh $(N=51)$, and $A=2.437$ and $D e_{\mathrm{cr}}=0.456$ (linear correlation coefficient, $r=0.997$ ) on the finer mesh $(N=101)$. In this way a more precise error measure based on the critical $D e$ value is $0.001 / 0.456=0.2 \%$, while it raises to $3.8 \%$ for the coefficient $A$.

\subsection{Effect of rounding corners: $R=0.05 d$ and $0.5 d$}

A natural question that arises is related with the presence of sharp corners and whether the bifurcation is triggered by some possible imbalance of the high stresses generated in those corner regions. Sharp corners are well known to be troublesome in the numerical solution of viscoelastic flow equations (Owens and Philips [22]) and in many of the early simulations of the cross-slot, in which only one quarter of the full geometry was simulated, the corner was artificially rounded by a slight amount (e.g. Remmelgas et al. [3]) in order to enable solutions at high Deborah numbers to be obtained. Even experimental studies (Schoonen et al. [5]) have followed the route of rounding the corners to avoid extreme stress concentration and allow easier interpretation of the birefringence visualizations. Since our argument to explain the bifurcation, and indeed the explanation put forth by Poole et al. [11], hinges on the high compressive stresses generated in the centreline region of the two incoming flow streams, then we do not expect rounding of the corners to affect significantly the bifurcation phenomenon. 

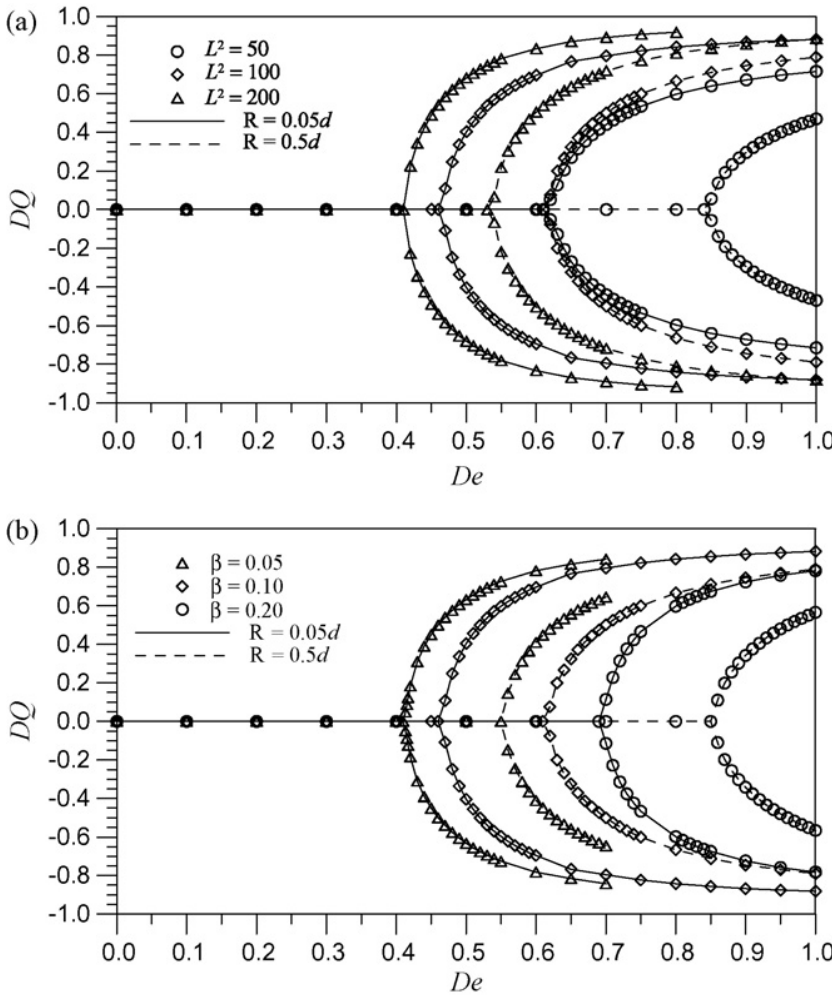

Fig. 9. Effect of rounding the corners on the asymmetry plot for two curvature radius with the FENE-CR model: $R=0.05 d$ and $0.5 d$. (a) $L^{2}=50,100$ and 200 and (b) $\beta=0.05$, $0.1,0.2$.

In order to test this hypothesis, two modified cross-slot geometries (see Fig. 1b) with corners rounded up to curvature radius of $0.05 d$ (small curvature) and 0.5d (large curvature) were prepared and covered with non-orthogonal meshes having similar characteristics to the sharp-corner geometries in Table 1 . The resulting critical Deborah numbers for the onset of the supercritical instability are provided in Tables 4 and 5 , for both a constant $\beta=0.1$ and increasing $L^{2}$, and a constant $L^{2}=100$ and increasing $\beta$; bifurcation plots are shown in Fig. 9 and the numerical data given in Table 6 for the milder curvature case. It is clear from these results that the above supposition holds: when the corners are first slightly rounded $(R=0.05 d)$ both the critical values and the variation of $D Q$ with $D e$ remains virtually unchanged from the sharp-corner cases. One may thus safely conclude that the triggering mechanism for the bifurcation is not controlled by near-corner features. For the larger curvature geometry the velocity field is significantly altered (as quantified in Fig. 10 for the base Newtonian flow) with an anticipated reduction of strain rate, $\dot{\varepsilon}=\partial u / \partial x$, on approaching the internal stagnation point at the cross-slot centre (by $32 \%$ from $R=0$ to $R=0.5 d$ ) and therefore the expected outcome is a stabilization of the flow with a delay of the instability onset, as we observe in our simulations and show in Table 5 and Fig. 11a. The change of the base flow strain rate is reflected in the extensional-related characteristics (the slope of the variation of $D e_{c r}$ with $L^{2}$ in Fig. 11a (solid lines), especially at low $L^{2}$, is now significantly different to the sharp-corner case) but the influence of concentration (dashed lines) is hardly different from the sharp-corner cases. In Fig. 11b we plot the same critical De data but as a function of the group $(1-\beta) L^{2} / \beta$ : a reasonable correlation is obtained thus implying that the critical Deborah number is in fact controlled by the ratio of polymer and solvent maximum elongational viscosities.
Table 6

Bifurcation data for the FENE-CR fluid in the rounded geometry $(R=0.5 d)$ : (a) varying $L^{2}(\beta=0.1)$ and (b) varying $\beta\left(L^{2}=100\right)$ (critical conditions indicated in bold)

\begin{tabular}{|c|c|c|c|c|c|}
\hline \multicolumn{2}{|c|}{ (a) $L^{2}=\mathbf{5 0}$} & \multicolumn{2}{|c|}{$L^{2}=\mathbf{1 0 0}$} & \multicolumn{2}{|c|}{$L^{2}=\mathbf{2 0 0}$} \\
\hline De & $D Q$ & De & $D Q$ & $D e$ & $D Q$ \\
\hline 0.00 & 0.000 & 0.00 & 0.000 & 0.00 & 0.000 \\
\hline 0.10 & 0.000 & 0.10 & 0.000 & 0.10 & 0.000 \\
\hline 0.20 & 0.000 & 0.20 & 0.000 & 0.20 & 0.000 \\
\hline 0.30 & 0.000 & 0.30 & 0.000 & 0.30 & 0.000 \\
\hline 0.40 & 0.000 & 0.40 & 0.000 & 0.40 & 0.000 \\
\hline 0.50 & 0.000 & 0.50 & 0.000 & 0.50 & 0.000 \\
\hline 0.60 & 0.000 & 0.60 & 0.000 & 0.53 & 0.000 \\
\hline 0.70 & 0.000 & 0.61 & 0.000 & 0.54 & 0.066 \\
\hline 0.80 & 0.000 & 0.62 & 0.076 & 0.55 & 0.218 \\
\hline 0.85 & 0.056 & 0.63 & 0.200 & 0.56 & 0.304 \\
\hline 0.86 & 0.138 & 0.64 & 0.268 & 0.57 & 0.370 \\
\hline 0.87 & 0.192 & 0.65 & 0.324 & 0.58 & 0.422 \\
\hline 0.88 & 0.234 & 0.66 & 0.372 & 0.59 & 0.468 \\
\hline 0.89 & 0.268 & 0.67 & 0.412 & 0.60 & 0.505 \\
\hline 0.90 & 0.296 & 0.68 & 0.444 & 0.65 & 0.638 \\
\hline 0.91 & 0.322 & 0.69 & 0.474 & 0.70 & 0.719 \\
\hline 0.92 & 0.346 & 0.70 & 0.501 & 0.75 & 0.772 \\
\hline 0.93 & 0.366 & 0.75 & 0.600 & 0.80 & 0.810 \\
\hline 0.94 & 0.384 & 0.80 & 0.666 & 0.85 & 0.836 \\
\hline 0.95 & 0.402 & 0.90 & 0.746 & 0.90 & 0.856 \\
\hline 1.00 & 0.470 & 1.00 & 0.790 & 1.00 & 0.884 \\
\hline \multicolumn{2}{|c|}{ (b) $\beta=0.05$} & \multicolumn{2}{|c|}{$\beta=\mathbf{0 . 1 0}$} & \multicolumn{2}{|c|}{$\beta=\mathbf{0 . 2 0}$} \\
\hline De & $D Q$ & $D e$ & $D Q$ & De & $D Q$ \\
\hline 0.00 & 0.000 & 0.00 & 0.000 & 0.00 & 0.000 \\
\hline 0.10 & 0.000 & 0.10 & 0.000 & 0.10 & 0.000 \\
\hline 0.20 & 0.000 & 0.20 & 0.000 & 0.20 & 0.000 \\
\hline 0.30 & 0.000 & 0.30 & 0.000 & 0.30 & 0.000 \\
\hline 0.40 & 0.000 & 0.40 & 0.000 & 0.40 & 0.000 \\
\hline 0.50 & 0.000 & 0.50 & 0.000 & 0.50 & 0.000 \\
\hline 0.55 & 0.000 & 0.60 & 0.000 & 0.60 & 0.000 \\
\hline 0.56 & 0.146 & 0.61 & 0.000 & 0.70 & 0.000 \\
\hline 0.57 & 0.244 & 0.62 & 0.076 & 0.80 & 0.000 \\
\hline 0.58 & 0.312 & 0.63 & 0.200 & 0.85 & 0.000 \\
\hline 0.59 & 0.366 & 0.64 & 0.268 & 0.86 & 0.110 \\
\hline 0.60 & 0.410 & 0.65 & 0.324 & 0.87 & 0.198 \\
\hline 0.61 & 0.448 & 0.66 & 0.372 & 0.88 & 0.256 \\
\hline 0.62 & 0.480 & 0.67 & 0.412 & 0.89 & 0.302 \\
\hline 0.63 & 0.510 & 0.68 & 0.444 & 0.90 & 0.342 \\
\hline 0.64 & 0.534 & 0.69 & 0.474 & 0.91 & 0.376 \\
\hline 0.65 & 0.558 & 0.70 & 0.500 & 0.92 & 0.406 \\
\hline 0.70 & 0.644 & 0.75 & 0.600 & 0.93 & 0.434 \\
\hline- & - & 0.80 & 0.666 & 0.94 & 0.458 \\
\hline- & - & 0.90 & 0.746 & 0.95 & 0.480 \\
\hline- & - & 1.00 & 0.790 & 1.00 & 0.566 \\
\hline
\end{tabular}

\subsection{Effect of viscoelastic model}

In terms of both shear and extensional properties the FENECR and FENE-P models are not too dissimilar, except for the shear viscosity which, as we have already discussed, is constant for the FENE-CR and shear-thinning for the FENE-P model. Since the cross-slot bifurcation is essentially controlled by extensional/compressional effects along the channels centreline, significant differences between these two models are not expected. Fig. 12 shows a comparison between the FENE-P (points denoted by symbols) and FENE-CR (lines) bifurcation plots revealing that the shear-thinning model tends to bifurcate at a lower Deborah number, for the two extensional parameters shown, that is $L^{2}=50$ and 100. At the higher extensibility the FENE-P model has a much stronger tendency to yield a non-steady end-state flow: while for the FENE-CR model the flow bifurcates but remains steady up to $D e \approx 1$, for the FENE-P model the results suggest that a slightly fluctuating bifurcated-flow regime tends to set in at much lower 


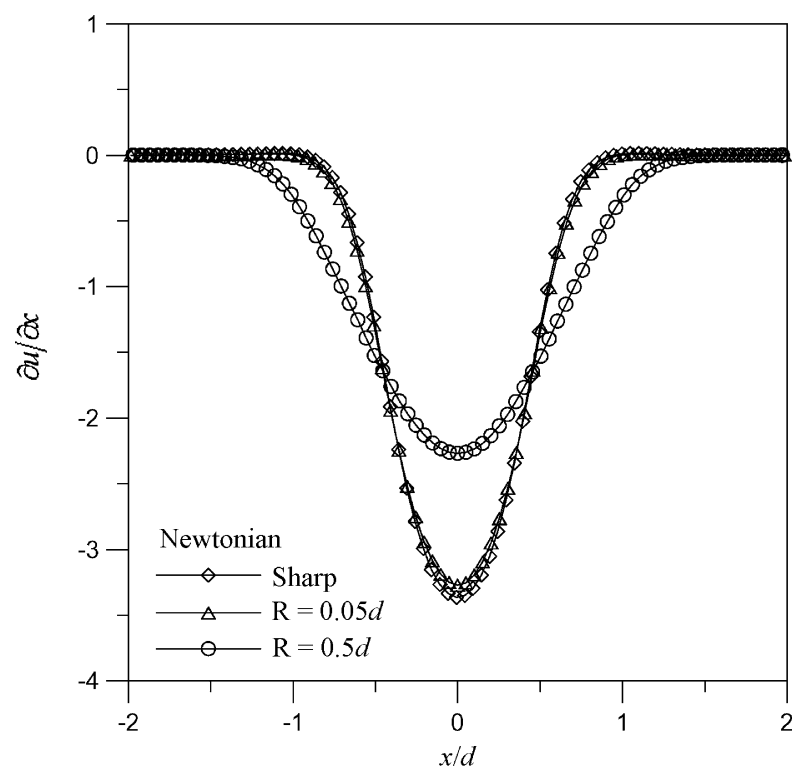

Fig. 10. Profiles of strain rate for Newtonian flow along the two incoming-channels centreline: influence of corner curvature, $R=0,0.05 d$ and $0.5 d$.

levels of Deborah number. In order to illustrate this unsteady phenomenon, which was also observed by Arratia et al. [9] in the microfluidic apparatus when this bifurcation was measured for the first time, the FENE-P model predictions of $D Q$ at $D e=0.46$ are
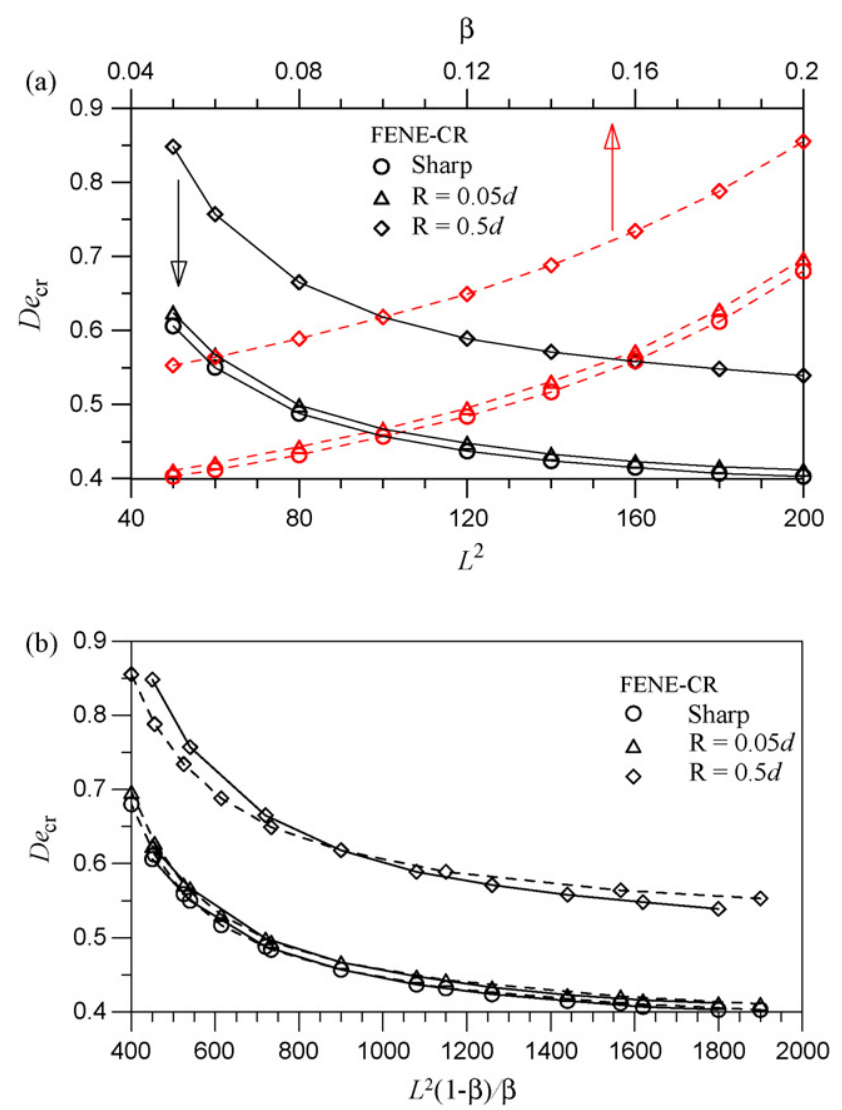

Fig. 11. Critical Deborah number for the FENE-CR model and various corner curvatures as a function of: (a) extensibility and polymer concentration separately and (b) the maximum extensional stress group $(1-\beta) L^{2} / \beta$. Note: in part (b) solid lines, $\beta=0.1$; dashed lines, $L^{2}=100$.

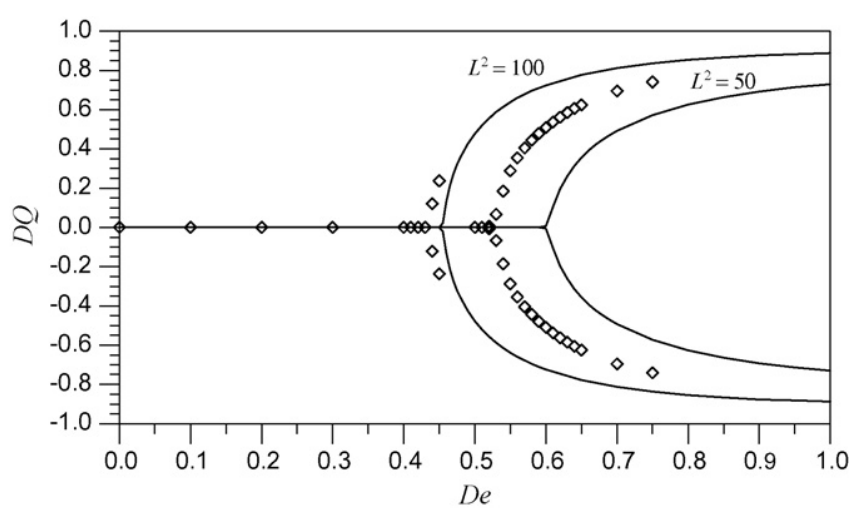

Fig. 12. FENE-P (symbols) and FENE-CR (full line) model predictions of the bifurcation diagram for $L^{2}=50$ and 100 .

plotted in Fig. 13 where a periodic evolution of the asymmetry is shown with a period of about 50 time units $(d / U)$. While this cyclic pattern is not an expected outcome of the FENE-P model, since in general shear-thinning tends to stabilize the numerical solution and allows higher Deborah numbers to be reached, the flow field resulting from the simulations in a zone very close to the reentrant corners shows signs of some local velocity perturbations, generated by the very large stresses existing at those locations, which appear to be responsible for the onset of the second instability and the time-dependent oscillations present in the numerical solution.

\subsection{Energy loss}

It would be useful to have an all-encompassing explanation for the onset mechanism that leads to the bifurcated flows observed in the cross-slot configuration for both constitutive models. In the limit, if one could establish that there is a reduction of energy to drive the flow when it bifurcates, then it would be possible to argue for a "less-effort" mechanism to explain the bifurcation. This effect is actually observed in simulation works as well as theoretical studies (Lagnado et al. [23]) and a possible measure of this effect is to evaluate the pressure drop required to drive the flow in the cross-

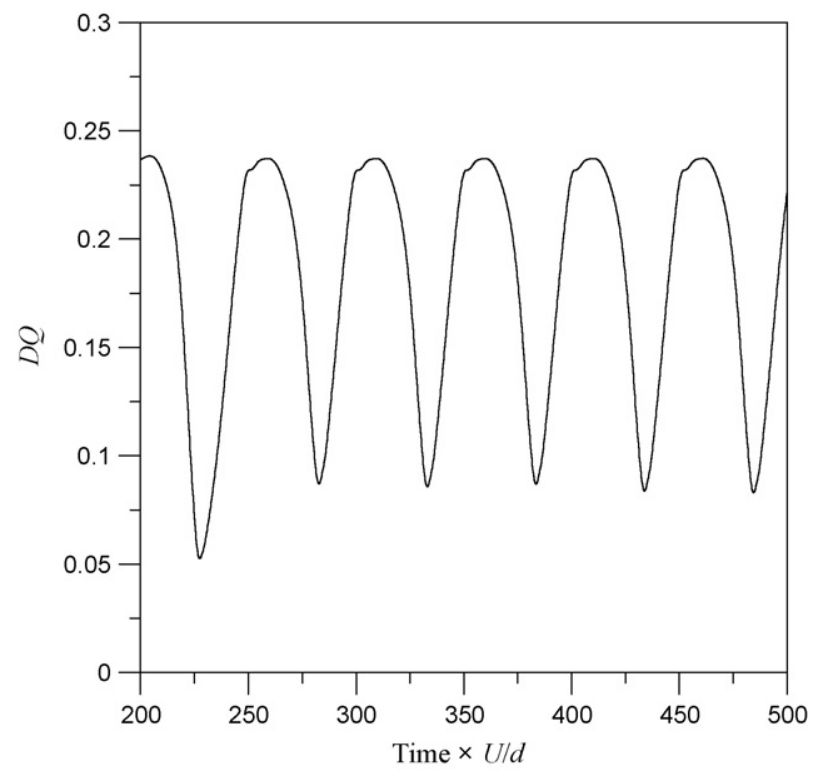

Fig. 13. Time-dependent evolution of $D Q$ for FENE-P model at $D e=0.46\left(L^{2}=100\right)$. 

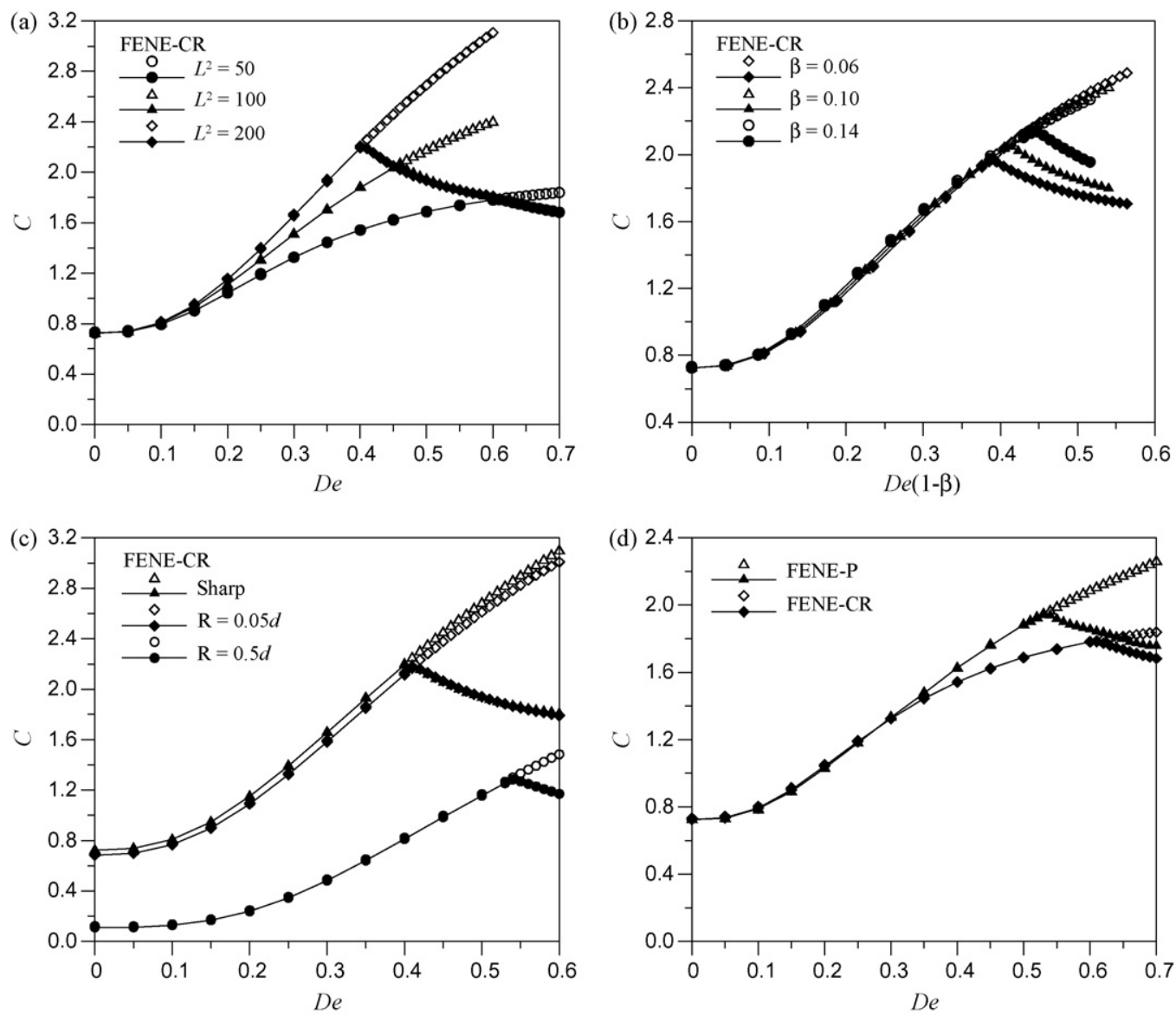

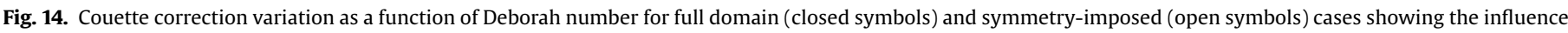

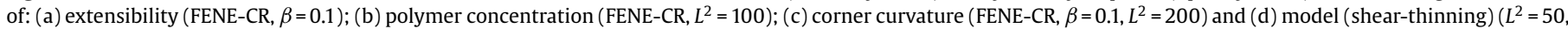
$\beta=0.1$ ).

slot configuration, as it is directly proportional to the energy loss. Fig. 14 shows the Couette correction $(C)$ for a number of situations, evaluated as the pressure drop between inlet and outlet after accounting for the fully developed pressure drop:

$C=\frac{\Delta p-\Delta p_{\mathrm{FD}}}{2 \tau_{\mathrm{W}}}$.

Here $\Delta p=p_{1}-p_{4}$ is the pressure difference between any of the inlets and one of the outlets (see Fig. 1), $\Delta p_{\mathrm{FD}}$ is the pressure drop required to drive fully developed flow in the inlet and outflow straight channels of width $d$ and total length $2 \times 10 d$ for an average velocity $U$, and $\tau_{\mathrm{w}}$ is the wall shear stress under fully developed conditions. As a consequence of the constant shear viscosity of the FENE-CR model, the wall shear stress is the same as for a Newtonian fluid with the same total viscosity $\eta_{0}$, that is $\tau_{\mathrm{w}}=3 \eta_{0} U /(d / 2)$. The fully developed pressure gradient is related to $\tau_{\mathrm{w}}$ by $-(\mathrm{d} p / \mathrm{d} x)_{\mathrm{FD}}=2 \tau_{\mathrm{w}} / d$, therefore $\Delta p_{\mathrm{FD}} /\left(\eta_{0} U / d\right)=2(L / d) \tau_{\mathrm{w}} /\left(\eta_{0} U / d\right)=240$. With the presence of shearthinning as in the FENE-P model both $\Delta p_{\mathrm{FD}}$ and $\tau_{\mathrm{w}}$ differ from the Newtonian case and although explicit analytical solutions can be derived (see Cruz et al. [20]) it is easier to re-interpret Eq. (9) as an excess pressure-gradient ratio, $C=(L / d)\left(\Delta p / \Delta p_{\mathrm{FD}}-1\right)$, since the previous relation between wall stress and pressure gradient is still valid.

For the base case with FENE-CR model, $L^{2}=100$ and $\beta=0.1$, the localised pressure loss is shown by the closed triangular symbols in Fig. 14a, while corresponding simulations in a quarter of the geom- etry, enforcing symmetry, results in the open triangular symbols. Whereas for $D e \leq D e_{\mathrm{cr}}=0.46$ the loss coefficient for those two calculations coincide, for $D e>D e_{\mathrm{cr}}$ the actual flow in the full geometry yields a reduced pressure loss in contrast to the symmetrical solution for which $C$ continues to rise with increasing $D e$. It is thus clear from Fig. 14a, and the other graphs in Fig. 14 where various influences other than extensibility are studied, that the bifurcation is accompanied by a reduction of the pressure loss, that is, less energy is required to drive the asymmetric flow compared with the corresponding "artificial" symmetric flow patterns. We also note that there are no signs of hysteresis effects: careful calculations starting from a bifurcated solution $\left(D e>D e_{\mathrm{cr}}\right)$ followed by small steps of decreasing De lead to a recovery of the symmetric pattern once $D e<D e_{\mathrm{cr}}$. Thus, regardless of the initial conditions, it was not possible to maintain an asymmetric pattern below the critical threshold. Fig. 14 may therefore be interpreted as a manifestation of a strict minimum energy rule, with two curves intersecting at $D e_{\mathrm{cr}}$, one for the symmetrical configuration and the other for the bifurcated one. When $D e$ is less than $D e_{\mathrm{cr}}$, the symmetric flow requires less consumption of energy and is preferred. When $D e$ exceeds $D e_{\mathrm{cr}}$ the opposite situation arises, with a smaller Couette correction for the full cross-slot geometry observed in Fig. 14, hence indicating that less energy is required to drive the asymmetric flow. Although this explanation is valid for the present inertialess viscoelastic flow, it is not a general principle as demonstrated by other steady instabilities exemplified by the Taylor-Couette cell pattern formation in the flow between co-axial rotating cylinders when 
the Taylor number (and consequently $R e$ ) exceeds a certain threshold.

Lagnado et al. [23] conducted a linear stability analysis of the Oldroyd-B model for the flow close to a planar stagnation point and found an instability at finite Weissenberg numbers. While their analysis can only be used as a guide to the present situation, since they assumed an unbounded flow with a base velocity field which is significantly different to the current case, it is interesting to note that they find a stronger stability for the case when the main stagnation streamlines meet at an acute angle. That is, a bifurcatedlike stagnation flow of an Oldroyd-B fluid is more stable than the configuration at $90^{\circ}$, which may be viewed as the corresponding un-bifurcated case. The theoretical results of Lagnado et al. [23] are corroborated by the present study: the bifurcated flow dissipates less energy and it is here preferred to the possible symmetric flow with incoming and outgoing streams making a $90^{\circ}$ angle.

In addition, Fig. 14 also illustrates the various influences of extensibility (Fig. 14a), polymer concentration (Fig. 14b), corner curvature (Fig. 14c) and constitutive model (Fig. 14d). While the maximum extensibility of the molecules for the FENE-CR model is seen (Fig. 14a) to have an important influence upon the localised pressure loss coefficient for Deborah numbers up to the critical point when the bifurcation occurs, with lower $L^{2}$ and thus smaller elongational viscosities showing reduced Couette corrections, once the asymmetrical patterns set in the evolution of $C$ vs. De is independent of extensibility. The solvent viscosity ratio, on the other hand, has hardly any influence upon $C$ under subcritical conditions, especially when plotted as a function of a modified Deborah number $D e(1-\beta)$, and the $C$ vs. $D e(1-\beta)$ variation for $D e>D e_{\mathrm{cr}}$ follows different paths essentially because for higher concentration (lower $\beta$ ) the bifurcation occurs at earlier De. These two graphs in Fig. 14 thus provide a clear distinction between two factors contributing to viscoelasticity: elongational-related elasticity and normal-stress elasticity. Fig. 14c shows once more that slightly rounding the corners has only a marginal effect on the flow and the pressure drop, while significant corner curvature strongly reduces the strain rates and hence gives rise to much smaller pressure drops in the whole range of $D e$, both sub- and supercritical. The final graph quantifies the effect on the constitutive model: for up to $D e \approx 0.35(W e \approx 1.0)$ both the FENE-CR and FENE-P yield the same values of $C$; however, for higher De the FENE-P predicts higher pressure losses. This might seem counter-intuitive on account of the shear-thinning characteristics of the FENE-P compared with the constant viscosity of the FENE-CR model. However it should be realised that the definition of $C$ balances the effect of shear-thinning due to the scaling with the wall shear stress ( $\tau_{\mathrm{w}}$ in the denominator of Eq. (9)); as commented above, $C$ might be written as a normalised additional channel length due to steeper pressure gradient compared with the fully developed case. In this case, the higher $C$ value of the FENE-P model must be related to a stronger rise of the elongational viscosity for this model which leads to the earlier transition to assymmetry observed before (cf. Fig. 12).

\section{Conclusions}

This numerical study has investigated the influence of finite extensibility and polymer concentration on the steady flow bifurcation that occurs in a cross-slot geometry having both sharp and rounded corners, with two different curvature radii. The rheological models employed were the FENE-CR and FENE-P, exhibiting a constant shear viscosity and a shear-thinning shear viscosity, respectively, and being adequate for the simulation of dilute unentangled polymer solutions. In all cases the inertialess viscoelastic flow through the symmetric cross-like geometry was predicted to become asymmetric, while remaining steady, for a Deborah number above a critical threshold level, in qualitative agreement with the experiments of Arratia et al. [9] in a microfluidic apparatus. That critical Deborah number decreases with increasing values of the extensibility parameter of the FENE models, here taken as $L^{2}$, thus suggesting the more elastic the fluid (in the sense of higher extensional viscosities) the earlier the flow destabilises and evolves to a bifurcated pattern. Regarding the influence of polymer concentration the effect is similar to that of $L^{2}$, which is not surprising since an added amount of polymer in a given solvent corresponds to a more elastic liquid. A measure of the amplitude of the secondary motion generated by the instability is provided by the asymmetry parameter $D Q$ which offers a measure of the imbalance of flow rate exiting through each outlet arm of the cross-slot. When the controlling variable $D e$ is raised, the bifurcation measure $D Q$ varies according to a theoretically expected square-root curve, given by Eq. (8), with the magnitude parameter $A$ depending only on the extensibility of the model $\left(L^{2}\right)$, while the critical Deborah number depends on both $L^{2}$ and $\beta$ which act jointly as $(1-\beta) L^{2} / \beta$. Finally, the introduction of a small degree of curvature into the cross-slot corners is shown to have practically no influence upon the bifurcation characteristics: both the amplitude $A$ and the critical Deborah $(D e)_{\mathrm{cr}}$ remain virtually unchanged. This finding provides support to the explanation of Poole et al. [11] for the bifurcation mechanism which resided on the compressive-like flow located around the central stagnation point.

\section{Acknowledgements}

Funding by Fundação para a Ciência e a Tecnologia (Portugal) under grant SFRH/BD/22644/2005 and projects PTDC/EMEMFE/70186/2006 and PTDC/EQU-FTT/71800/2006 are gratefully acknowledged. We are grateful to a referee for suggesting the scaling used in Fig. 11b.

\section{References}

[1] O.G. Harlen, J.M. Rallison, M.D. Chilcott, High-Deborah-number flows of dilute polymer solutions, J. Non-Newtonian Fluid Mech. 34 (1990) 319-349.

[2] P. Singh, L.G. Leal, Finite element simulation of flow around a $3 \mathrm{pi} / 2$ corner using the FENE dumbbell model, J. Non-Newtonian Fluid Mech. 58 (1995) 279-313.

[3] J. Remmelgas, P. Singh, L.G. Leal, Computational studies of nonlinear dumbbell models of Boger fluids in a cross-slot flow, J. Non-Newtonian Fluid Mech. 88 (1999) 31-61.

[4] G.W.M. Peters, J.F.M. Schoonen, F.P.T. Baaijens, H.E.H. Meijer, On the performance of enhanced constitutive models for polymer melts in a cross-slot flow, J. Non-Newtonian Fluid Mech. 82 (1999) 387-427.

[5] J.F.M. Schoonen, F.H.M. Swartjes, G.W.M. Peters, F.P.T. Baaijens, H.E.H. Meijer, A 3D numerical/experimental study on a stagnation flow of a polyisobutylene solution, J. Non-Newtonian Fluid Mech. 79 (1998) 529-561.

[6] W.M.H. Verbeeten, G.W.M. Peters, F.P.T. Baaijens, Viscoelastic analysis of complex polymer melt flows using the eXtended Pom-Pom model, J. NonNewtonian Fluid Mech. 108 (2002) 301-326.

[7] J. Remmelgas, L.G. Leal, Computational studies of the FENE-CR model in a tworoll mill, J. Non-Newtonian Fluid Mech. 89 (2000) 231-249.

[8] J. Feng, L.G. Leal, Transient extension and relaxation of a dilute polymer solution in a four-roll mill, J. Non-Newtonian Fluid Mech. 90 (2000) 117-123.

[9] P.E. Arratia, C.C. Thomas, J. Diorio, J.P. Gollub, Elastic instabilities of polymer solutions in cross-channel flow, Phys. Rev. Lett. 96 (2006) 144502.

[10] R.G. Larson, Review: instabilities in viscoelastic flows, Rheol. Acta 31 (1992) 213-263.

[11] R.J. Poole, M.A. Alves, P.J. Oliveira, Purely elastic flow asymmetries, Phys. Rev. Lett. 99 (2007) 164503.

[12] R.B. Bird, O. Hassager, R.C. Armstrong, C.F. Curtiss, Dynamics of Polymeric Liquids, Kinetic Theory, vol. 2, John Wiley, New York, 1987.

[13] R.B. Bird, P.J. Dotson, N.L. Johnson, Polymer solution rheology based on a finitely extensible bead-spring chain model, J. Non-Newtonian Fluid Mech. 7 (1980) 213-235.

[14] M.D. Chilcott, J.M. Rallison, Creeping flow of dilute polymer solutions past cylinders and spheres, J. Non-Newtonian Fluid Mech. 29 (1988) 381-432.

[15] J.G. Oldroyd, On the formulation of rheological equations of state, Proc. R. Soc. Lond. A 200 (1950) 523-541. 
[16] P.J. Oliveira, F.T. Pinho, G.A. Pinto, Numerical simulation of on-linear elastic flows with a general collocated finite-volume method, J. Non-Newtonian Fluid Mech. 79 (1998) 1-43.

[17] M.A. Alves, P.J. Oliveira, F.T. Pinho, A convergent and universally bounded interpolation scheme for the treatment of advection, Int. J. Numer. Meth. Fluids 41 (2003) 47-75.

[18] P.J. Oliveira, Method for time-dependent simulations of viscoelastic flows: vortex shedding behind cylinder, J. Non-Newtonian Fluid Mech. 101 (2001) 113-137.

[19] P.J. Oliveira, Asymmetric flows of viscoelastic fluids in symmetric planar expansion geometries, J. Non-Newtonian Fluid Mech. 114 (2003) 33-63.
[20] D.O.A. Cruz, F.T. Pinho, P.J. Oliveira, Analytical solutions for fully developed laminar flow of some viscoelastic liquids with a Newtonian solvent contribution, J. Non-Newtonian Fluid Mech. 132 (2005) 28-35.

[21] G.N. Rocha, R.J. Poole, P.J. Oliveira, Bifurcation phenomena in viscoelastic flows through a symmetric 1:4 expansion, J. Non-Newtonian Fluid Mech. 141 (2007) $1-17$.

[22] R.G. Owens, T.N. Phillips, Computational Rheology, World Scientific Press, 2002.

[23] R.R. Lagnado, N. Phan-Thien, L.G. Leal, The stability of two-dimensional linear flows of an Oldroyd-type fluid, J. Non-Newtonian Fluid Mech. 18 (1985) 25-59. 